\title{
Rich Phase Separation Behavior of Biomolecules
}

\author{
Yongdae Shin ${ }^{1,2, *}$
}

\author{
${ }^{1}$ Department of Mechanical Engineering, Seoul National University, Seoul 08826, Korea, ${ }^{2}$ Interdisciplinary Program in \\ Bioengineering, Seoul National University, Seoul 08826, Korea \\ *Correspondence: ydshin@snu.ac.kr \\ https://doi.org/10.14348/molcells.2021.0204 \\ www.molcells.org
}

Phase separation is a thermodynamic process leading to the formation of compositionally distinct phases. For the past few years, numerous works have shown that biomolecular phase separation serves as biogenesis mechanisms of diverse intracellular condensates, and aberrant phase transitions are associated with disease states such as neurodegenerative diseases and cancers. Condensates exhibit rich phase behaviors including multiphase internal structuring, noise buffering, and compositional tunability. Recent studies have begun to uncover how a network of intermolecular interactions can give rise to various biophysical features of condensates. Here, we review phase behaviors of biomolecules, particularly with regard to regular solution models of binary and ternary mixtures. We discuss how these theoretical frameworks explain many aspects of the assembly, composition, and miscibility of diverse biomolecular phases, and highlight how a model-based approach can help elucidate the detailed thermodynamic principle for multicomponent intracellular phase separation.

Keywords: condensate, membrane-less organelle, phase diagram, phase separation, regular solution model

\section{INTRODUCTION}

Cells carry out highly sophisticated tasks such as self-repair, migration, differentiation and division. These behaviors emerge from collective interactions between biomolecules as well as complex biochemical reaction networks. The robust- ness of cellular processes is especially remarkable when considering high molecular diversity and abundance found in the cell. The cell interior is highly crowded with numerous protein species present at an estimated concentration around $3 \mathrm{mM}$ (Milo, 2013). A daunting task cells face is to coordinate various intracellular processes which take place simultaneously in the confined three-dimensional space. Cells address this seemingly chaotic situation, at least partially, through compartmentalization.

Eukaryotic cells contain numerous compartments or organelles that consist of a specific set of biomolecules and perform specialized biological functions. In addition to membrane-bound organelles such as nucleus and mitochondria, cells also harbor those without encapsulating membranes. Examples fall into the latter category include, among others, nucleoli, nuclear speckles, paraspeckles, promyelocytic leukemia (PML) bodies in nucleus and cytoplasmic stress granules, germ granules and P bodies (Buchan and Parker, 2009; Chujo and Hirose, 2017; Mao et al., 2011). These membrane-less organelles, recently termed condensates, have several interesting biophysical properties (Banani et al., 2017; Shin and Brangwynne, 2017). First, even in the absence of physical barriers such as lipid membranes, they can still enrich a set of biomolecules within them. Specific marker proteins are recruited into condensates, enabling the visualization of condensates using fluorescence microscopy (Thul et al., 2017). Second, biomolecules in these organelles are not organized in a well-defined three-dimensional arrangement, which is an important feature distinguishing them from macromolecular complexes such as ribosome and RNA polymerases of which

Received 1 August, 2021; revised 10 September, 2021; accepted 10 September, 2021; published online 30 December, 2021 
individual subunits make tight contacts with one another in a designated stoichiometry and orientation (Freeman Rosenzweig et al., 2017; Wilfling et al., 2020). Moreover, condensates such as nucleoli and stress granules tend to be much larger than typical macromolecular complexes, reaching up to a few microns in size. Third, internal components of condensates are not completely confined inside the organelles, as examined with experimental techniques such as fluorescence recovery after photobleaching. Although the degree of molecular mobility varies depending on the type of biomolecules and the physical state of condensates, components often exhibit diffusive mobility within the organelle and also undergo dynamic exchange with the surrounding cellular space (Chen and Huang, 2001; Mollet et al., 2008; Weidtkamp-Peters et al., 2008). These data collectively suggest that membrane-less compartments are not a mere aggregate of biomolecules. Rather, they represent a condensed state of living matter where constituent biomolecules are cohesively held together by intermolecular interactions that constantly break and reform.

Recently, a biophysical principle driving the formation of membrane-less organelles has begun to be uncovered. In 2009, P granules in Caenorhabditis elegans embryo were shown to exhibit liquid-like material properties (Brangwynne et al., 2009). When the one-cell embryo divides, $P$ granules spatially segregate into one of daughter cells, a process implicated in germline specification. Quantitative image analysis of the segregation process revealed that the segregation was not a result of transport of intact $P$ granules to one side of the cell, but rather a combined outcome of localized condensation and dissolution. Moreover, when cytoplasmic flow was induced, $P$ granules that initially wet the surface of nuclei became detached and exhibited dripping as well as fusion, a hallmark of liquid states. A later work showed that the nucleoli of Xenopus laevis oocytes had round morphology and upon fusing one another, the dumbbell-like shape gradually relaxed back to round one (Brangwynne et al., 2011). These behaviors are again consistent with liquid-like nature displaying surface tension which drives the system toward the minimum of surface area. The presence of liquid-like organelles within cyto- or nucleoplasm immediately suggests that liquid-liquid phase separation can be a physiochemical principle for the biogenesis of membrane-less organelles. Numerous works afterwards have shown that phase separation indeed plays a key role in organizing intracellular space (Li et al., 2012; Molliex et al., 2015; Nott et al., 2015; Patel et al., 2015) and the list of biological processes involving phase separation is continuously expanding (Feric et al., 2016; Franzmann et al., 2018; Sabari et al., 2018; Strom et al., 2017; Su et al., 2016; Yasuda et al., 2020; Zeng et al., 2016; Zhang et al., 2018).

In this paper, we review fundamentals of phase separation processes with a primary focus on diverse phase diagrams exhibited by biomolecular phase separation. We begin our discussion with the regular solution model of a binary mixture composed of one type of solute dissolved in solvent. This simple model explains a key aspect of phase separation such as the concentration-dependent assembly. We then build up complexity by considering a ternary regular solution model with additional type of solute. Special emphasis is given to rich behaviors the ternary phase-separating systems can exhibit. We discuss how intermolecular interactions can define the specific shape of phase diagrams of the ternary mixture. In doing so, we motivate a model-based approach in analyzing experimental data and designing new experiments that can be useful in gaining new insights into the phase behaviors of cells, an inherently multicomponent system.

\section{PHASE SEPARATION IN BINARY MIXTURES}

Phase separation is commonly encountered in everyday experience in mixtures such as oil-water, oil-vinegar, and metal alloy systems. The simplest model capturing the essence of phase separation is a lattice-based regular solution model for a binary mixture, also sometimes called Flory-Huggins model (Rubinstein and Colby, 2003). The model deals with a lattice of which individual site is filled with either solute or solvent species (Fig. 1A). The volume fraction of the solute, the number of solutes divided by the total number of lattice sites, is a major parameter that can be controlled as an input in the model. The phase behavior of the binary regular solution model is dictated by the free-energy of mixing, comprising both entropic as well as energetic contributions (Brangwynne et al., 2015; Posey et al., 2018). In the absence of any interactions between species, the solutes stay in a well-mixed state without any phase separation (Figs. 1A and 1B). This is because the system can minimize free energy by maximizing possible configurations, equivalently entropy, in the wellmixed state. Energetic interactions between species can either promote or impede mixing. In the regular solution model, interaction energies between only nearest neighbors are taken into consideration under mean-field approximation. When homotypic interactions are favored over heterotypic ones, the binary system can decrease its free energy through phase separation, dividing system volume into two regions with distinct compositions (Figs. 1A and 1B).

These conditions for phase separation can be conveniently expressed in the form of a phase diagram where the $X$ and $Y$ axis typically represent the volume fraction or concentration of phase-separating solutes and temperature, respectively. In the phase diagram of binary systems, a curved line called binodal or coexistence curve delimits a single-phase versus a two-phase region (Fig. 1C). Thus, for a given temperature and solute type (a fixed $Y$ value in the phase diagram), there exists a concentration threshold, called saturation concentration, above which phase separation is induced (Fig. 1D). Phase separation divides the system volume into two distinct regions, a dense phase with a high concentration of solutes and a dilute phase with low concentration. The concentration of solutes within each phase is again specified in the phase diagram: the dilute phase concentration is fixed to saturation concentration and the concentration in the dense phase is given by the right-hand side of binodals (Fig. 1D). Importantly, as the system enters deeper into the two-phase region, the model states that solute concentrations in each phase do not change, but the volume of the dense phase increases. The degree of phase separation can be also modulated by changing either temperature or interaction strengths be- 
A

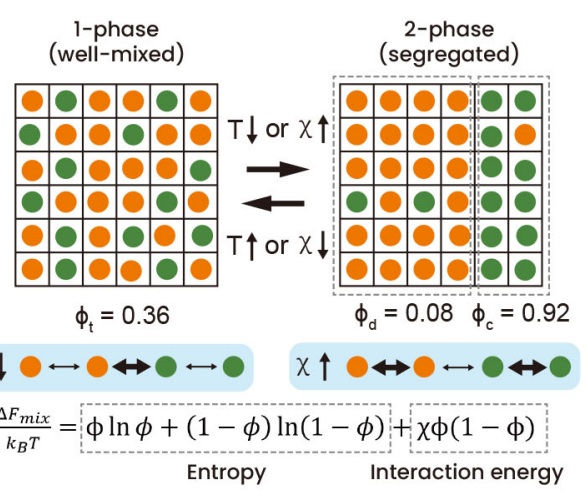

B

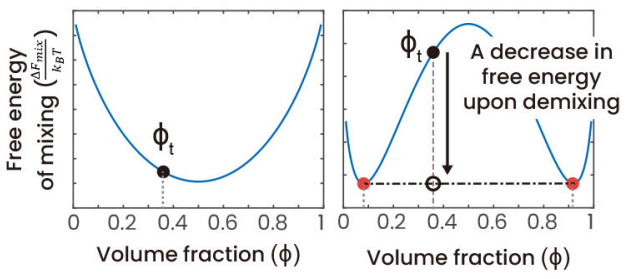

C
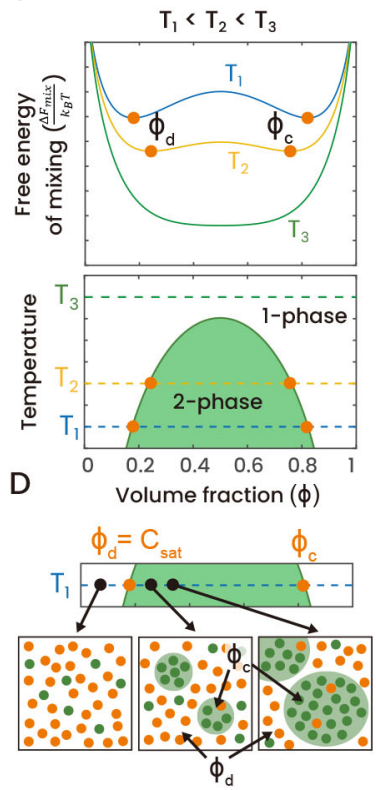

$E$

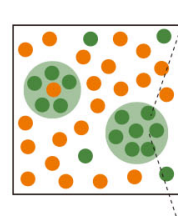

Intrinsically disordered regions (IDRs)

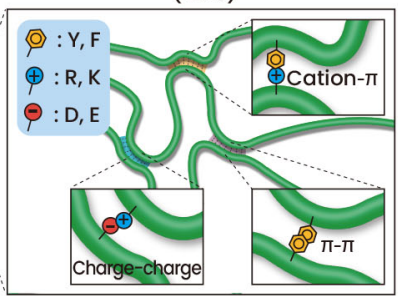

$F$

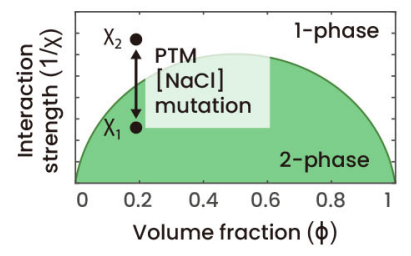

Fig. 1. Binary regular solution model and its phase behaviors. (A) A binary regular solution model. In the binary system, two types of species, a solute and a solvent, are distributed in each lattice site. The volume fraction of solutes, $\phi$, serves as an important parameter for describing the state of the system. When the system undergoes phase separation into the two-phase state, the system volume is divided into two regions with different volume fractions of solutes. As an example, this figure shows a transition between the single-phase state with a total solute volume fraction, $\phi_{\mathrm{t}}$ and the two-phase state with volume fractions $\phi_{\mathrm{d}}$ and $\phi_{\mathrm{c}}$. Interactions between species are characterized by the Flory interaction parameter, $\chi$. When homotypic interactions are favored over heterotypic ones, phase separation can be induced. The free energy of mixing per unit volume, $\Delta \mathrm{F}_{\text {mix }}$, is given by the sum of entropic contribution and interaction energies. (B) (Left) In the absence of any interactions between species $(\chi=0)$, the compositional dependence of the free energy is convex, leading to the well-mixed single-phase state. (Right) When homotypic interactions are strong enough, the free energy curve becomes concave, and the system can lower the free energy by dividing the system volume into two regions with differing compositions. The solid and empty black circles denote the total free energy of the system before and after phase separation, respectively. Red circles represent solute volume fractions in the dilute and dense phases. (C) Temperature dependence of the free energy and the resulting phase diagram. (Top) For each temperature, the free energy curve determines whether phase separation occurs or not and, if it occurs, volume fractions in two phases $\left(\phi_{d}\right.$ and $\left.\phi_{c}\right)$ as well. (Bottom) Phase diagrams are typically drawn in the temperature-volume fraction (concentration) plane. (D) In the binary regular solution model, at constant temperature, increasing solute concentration increases the volume of the dense phase while the solute concentrations in two coexisting-phases are fixed to values at the phase boundary. Frequently, a concentration corresponding to the left-hand side boundary of the coexistence region is called the saturation concentration, $C_{\text {sat. }}$. $(E)$ Proteins containing intrinsically disordered regions can often phase-separate through homotypic IDR-IDR interactions mediated by charged and/or aromatic residues. (F) The phase diagram of the binary regular solution model can also be plotted in the concentration-interaction parameter plane. Changes in interaction strengths, caused by perturbations such as post-translational modifications, mutations, and varying salt concentrations, can modulate phase separation behavior.

tween species. For example, at higher temperature (or when homotypic interactions become less favorable), the saturation concentration increases, and thus higher concentrations of solutes are required for phase separation. Taken together, the regular solution theory provides several detailed features that can be used to test whether binary phase separation takes place in the system being probed.

Liquid-liquid phase separation of a binary mixture of protein and aqueous solution has been observed at least as early as the 1970s (Ishimoto and Tanaka, 1977). For lysozyme and lens crystallin proteins, temperature-dependent phase separation behaviors were observed, and their coexistence curves were quantitatively measured (Broide et al., 1991; Thomson et al., 1987). The phase behaviors of these proteins are studied in relation to Cataract diseases (Benedek, 1997). More recently, numerous studies have reported binary phase separation of proteins harboring intrinsically disordered regions (IDRs) (Brangwynne et al., 2015; Uversky, 2021). IDRs represent polypeptide segments lacking well-defined three-dimensional folding (Hong et al., 2020). Their sequences tend to be simple in composition, and thus IDRs are closely related to low-complexity domains (Tompa, 2012). Recently, phase separation of IDR-containing proteins (IDPS) has attracted massive attention since many components of condensates, particularly RNA- and DNA-binding proteins, are known to harbor IDRs (Kato et al., 2012; Uversky, 2017). Purified IDPs 
undergo phase separation to form dense liquid droplets (Lin et al., 2015; Molliex et al., 2015; Patel et al., 2015). Notably, several features predicted from the regular solution model have been indeed observed, including the presence of saturation concentrations and the characteristic temperature-dependence of phase separation (Burke et al., 2015; Molliex et al., 2015; Nott et al., 2015). In particular, an in-vitro work on LAF-1, a P-granule component, demonstrated that the LAF-1 droplets are in equilibrium with a saturated protein solution outside of droplets by showing that the protein concentrations in the dilute phase are fixed at the saturation concentration even when total protein concentrations vary (Elbaum-Garfinkle et al., 2015).

Weak multivalent interactions mediated by residues along polypeptide chains are thought to be important for phase separation of IDPs, as often modeled with the sticker-spacer framework (Choi et al., 2020) (Fig. 1E). Stickers are a segment of polypeptides exhibiting attractive interactions with other stickers, acting as a key element driving phase separation. Spacers link neighboring stickers and play a modulatory role in phase separation (Harmon et al., 2017). Specific residues corresponding to stickers vary depending on the type of interactions biomolecules exhibit. Diverse interaction modes including electrostatic (Nott et al., 2015; Pak et al., 2016), cation-pi (Wang et al., 2018), and hydrophobic interactions (Murthy et al., 2019; Simon et al., 2017) have been shown to play key roles in promoting phase separation (Dignon et al., 2020). The regular solution model suggests that altering intermolecular interactions can impact phase separation of solutes (Fig. 1F). Indeed, screening electrostatic interactions using salt ions leads to a decrease in droplet sizes and eventual dissolution for electrostatically-driven condensates (Elbaum-Garfinkle et al., 2015; Nott et al., 2015; Zamudio et al., 2019). Interactions between stickers are also highly affected by mutations and post-translational modifications (PTMs), which can ultimately cause a significant change in phase behaviors (Hofweber et al., 2018; Monahan et al., 2017; Qamar et al., 2018; Ryan et al., 2018; Snead and Gladfelter, 2019). Moreover, cells utilize PTMs of condensate components as a regulatory mechanism for condensate assembly (Rai et al., 2018). Notably, although the regular solution model is successful in describing the overall phase separation properties of binary mixtures, a more advanced theory in combination with computer simulation is often required for dissecting quantitative aspects of phase behaviors such as the sequence-specific locations of the coexistence region (Lin et al., 2016; Wei et al., 2017).

For living cells with numerous types of biomolecules, the binary regular solution model appears to be over-simplified. However, several works have shown that the model still provides a biophysical explanation for key features of condensate assembly. Nucleoli in C. elegans are shown to assemble only when the concentration of nucleolar protein FIB-1 reaches a concentration threshold, consistent with the presence of the saturation concentration (Weber and Brangwynne, 2015). A recent live cell reconstitution work also shows that stress granules assemble with a clear concentration threshold for G3BP (Sanders et al., 2020). DDX4 is one of model IDPs of which phase behaviors are extensively studied (Nott et al.,
2015; 2016). When expressed in cells, the DDX4 IDR concentration in dilute phase is maintained at a similar level even though the total protein concentration increases over an order of magnitude (Klosin et al., 2020), an effect fully consistent with the binary regular solution model. This behavior suggests that condensates can function to buffer protein expression noise. Similar behaviors of concentration thresholding are also observed for light-inducible systems such as optoDroplets (Shin et al., 2017; Wei et al., 2020) and Corelets (Bracha et al., 2018). Since the degree of super-saturation can be precisely defined using external light, these systems are particularly useful to quantitatively study phase separation in living cells (Basu et al., 2020; Zhu et al., 2019).

\section{RICH PHASE BEHAVIORS OF TERNARY SYSTEMS}

Adding another type of solute into the system leads to drastically richer phase behaviors, as hinted by a ternary regular solution model (Deviri and Safran, 2021; Dignon et al., 2020; Meijering, 1950). With additional components in the system, the phase diagram now incorporates another axis for the concentration of the second solute, and becomes three-dimensional (Fig. 2A). Typically, concentrations of coexisting phases are described by a two-dimensional slice at a given temperature, and thus phase diagrams of ternary systems are usually plotted in the concentration-concentration plane. Here, we consider five different scenarios of inter-species interactions, each of which leads to phase diagrams of distinct shape and molecular partitioning.

The first scenario involves two types of solutes which exhibit strong heterotypic interactions but not homotypic ones (Fig. 2B). Since each solute does not undergo self-association, a solution with only one type of solute stays in a well-mixed state. However, when both types of solutes are present, attractive heterotypic attractions can lead to phase separation. Thus, a phase diagram for this type of system typically forms a closed loop detached from both axes. When the solute concentrations fall in the coexistence region enclosed by the loop, phase separation occurs to generate two coexisting phases: a dense and a dilute phase. The concentrations of solutes in each phase are generally given by tie-lines. The tieline of the system in the first scenario has a positive slope, indicating that both solutes are enriched in the dense phase and depleted in the dilute phase. A mixture of polycation and polyanion, such as positively-charged polypeptides and RNA, is a classic example in this category (Aumiller and Keating, 2016; Banerjee et al., 2017; Ukmar-Godec et al., 2019). More recently, pairs of tandem protein repeats with interacting modules, such as $\mathrm{PRM}_{x}-\mathrm{SH}_{Y}, \mathrm{SUMO}_{x}-\mathrm{SIM}_{Y}$, and $\mathrm{pY} \mathrm{Y}^{-}$ $\mathrm{SH}_{Y}$ ( $X$ and $Y$ are the number of repeats), have been shown to play important roles in intracellular phase separation (Banani et al., 2016; Li et al., 2012). These multivalent proteins are found in various cell signaling pathways including $T$ cell activation (Su et al., 2016) and neuronal synapses (Chen et al., 2020). Concentrating reaction components within condensed liquid droplets is shown to enhance biochemical reactions such as actin assembly (Su et al., 2016) as well as enzymatic activity (Peeples and Rosen, 2021).

The second case is a mixture of a self-associating solute 
A
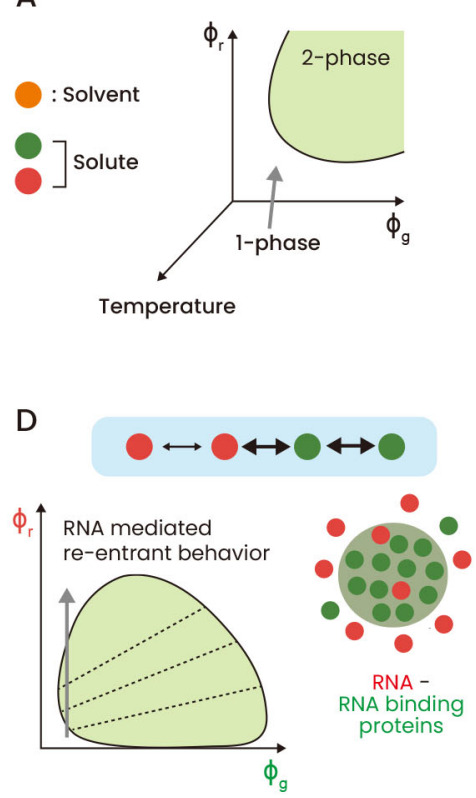

B

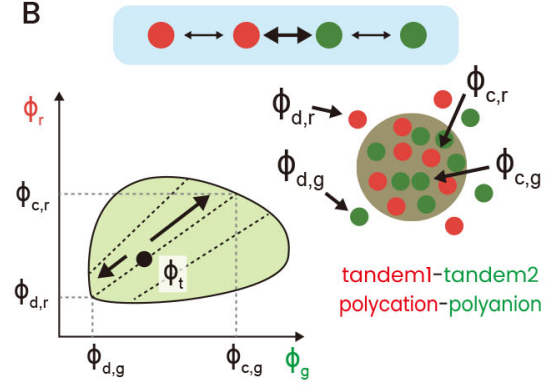

$\mathrm{E}$

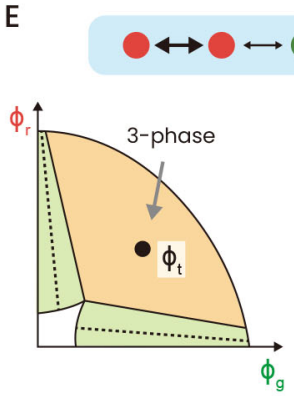

C

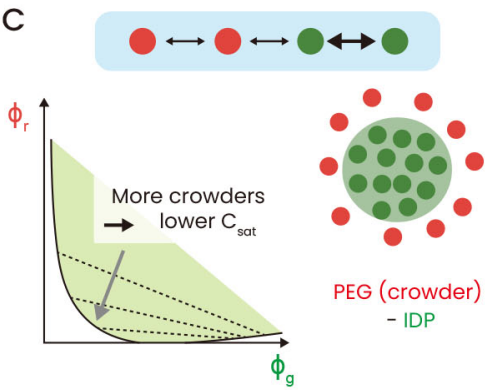

$\mathrm{F}$

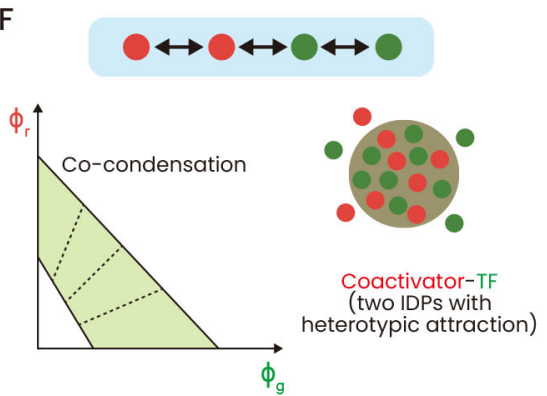

Fig. 2. Diverse phase behaviors of ternary systems. (A) With an additional type of solute, the phase diagram of ternary systems becomes three-dimensional, but a two-dimensional slice in the plane of two solute concentrations is typically used. (B) A mixture of two solutes with attractive heterotypic interactions. Examples include pairs of polycation-polyanion or multivalent proteins with tandem modular interaction domains. Each solute alone cannot phase-separate, but addition of the interacting partners can induce phase separation. Throughout Fig. 2, the dotted lines represent tie-lines which describe the direction of phase separation. The points where tie-lines meet the coexistence curve (a loop in the figure) give solute concentrations in coexisting phases. (C) A mixture of a phase-separating solute and a crowder. Increasing the concentration of crowders leads to a decrease in the saturation concentration of the phase-separating solute. Crowders promote phase separation through an excluded volume effect. In this case, the tie-lines are oriented negatively, indicating that the crowders are excluded from the dense phase. (D) A mixture of scaffold-client solutes. The scaffold (green species) is a type of solute that can undergo phase separation alone, and the client (red species) exhibits attractive heterotypic interactions with the scaffold. This feature is reflected in the phase diagram: the 2-phase coexistence region intersects the X-axis corresponding to the absence of clients. Examples of this category include RNA and RNA binding proteins. As clients, RNA enhances phase separation of RNA binding proteins when present at low amounts, but at higher concentrations it ultimately causes dissolution of dense phases. (E) When two solutes exhibit attractive homotypic interactions but minimal heterotypic ones, three distinct phases can coexist. In this case, there are two immiscible dense phases, each of which is primarily enriched with one type of solute. (F) If attractive heterotypic interactions are as strong as homotypic ones, droplets of two solutes become miscible, forming a single dense phase enriched with both types of solutes. An example includes co-condensation of transcription factors (TFs) and coactivators.

and the other one exhibiting neither homo- nor heterotypic interactions. The self-associating solute can phase-separate even in the absence of the other solute, but the latter can promote phase separation through excluded volume interactions (i.e., two types of solutes cannot occupy the same volume simultaneously). Thus, the inert solute acts as a crowder. Typically, the coexistence region in this case intersects one of the axes, corresponding to the self-associating solute, and tielines are oriented with a negative slope (Fig. 2C). Thus, the dense phase consists mostly of the phase-separating solute, devoid of crowders. Examples in this scenario include purified IDPs in the presence of crowding reagents such as PEG or dextran (Cai et al., 2019; Lin et al., 2015; Sabari et al., 2018). Increasing the concentration of crowders further promotes phase separation, consistent with a decrease in the saturation concentration (Ray et al., 2020; Zhang et al., 2020). For IDPs with weaker homotypic interactions, crowders are required for phase separation (Kanaan et al., 2020; Patel et al., 2015; Sabari et al., 2018). The phase diagram for these proteins can also be described with a similar coexistence region but detached from the axis for the self-associating solute. The cell interior is highly crowded with biomolecules that do not undergo any specific interactions with many others. The crowded intracellular environment is likely to play a similar role in enhancing biomolecular phase separation.

Adding attractive heterotypic interactions to the second scenario, particularly relevant in the context of phase separation control in the cell is a mixture of a self-associating scaffold solute and a client solute exhibiting heterotypic interactions but minimal homotypic ones. Although the detailed modulatory effects of clients on scaffold phase behavior vary depending on the exact nature of scaffold-client interactions (Ruff et al., 2021), the ternary model predicts that, unlike crowders, client solutes can function in two opposite man- 
ners. When a small amount of client solutes is around, phase separation of scaffolds is enhanced due to additional crosslinks clients provide (Fig. 2D). However, an excess of clients can inhibit phase separation since interactions between clients and scaffolds begin to interfere with scaffold-scaffold interactions. This effect has been observed in a mixture of RNA and RNA binding proteins. RNA binding proteins often harbor positively charged IDRs such as RGG motifs together with various RNA recognition motifs (Wiedner and Giudice, 2021). Interactions between these motifs and RNA can strengthen a network of inter-molecular interactions driving phase separation. Indeed, multiple works have shown that RNA promotes phase separation of RNA binding proteins such as FUS, hnRNPA1, and G3BP1 (Guillén-Boixet et al., 2020; Maharana et al., 2018; Molliex et al., 2015; Yang et al., 2020). However, further increasing RNA concentrations begins to adversely affect phase separation, leading to shrinkage of protein droplets and eventual dissolution (Maharana et al., 2018). This "re-entrant phase behavior" plays an important regulatory role in modulating biomolecular phase separation in living cells (Banerjee et al., 2017). A recent work shows that during transcription, initial RNA production can enhance phase separation of transcriptional machineries such as MED1 coactivator, which further promotes transcription (Henninger et al., 2021). However, local accumulation of RNA then causes dissolution of transcriptional condensates. This RNA-mediated feedback control mechanism helps explain the origin of long-observed transcriptional bursts, a stochastic RNA production interspersed between periods of inactivity. The solubilizing effect of RNA also seems to play a role in maintaining protein homeostasis. Prion-like RNA binding proteins can undergo aberrant phase transitions from liquid droplets to solid aggregates (Lin et al., 2015; Molliex et al., 2015; Murakami et al., 2015; Patel et al., 2015), a process implicated in the onset of various neurodegenerative diseases (Mathieu et al., 2020). Disease-causing mutations are often found to impair nuclear-cytoplasmic trafficking, leading to the accumulation of mutated proteins in cytoplasmic aggregates (Dormann et al., 2010). It has been shown that a high concentration of nuclear RNA can maintain prion-like RNA binding proteins in the soluble state, preventing unwanted aberrant transitions into solid aggregates (Maharana et al., 2018).

When both solutes exhibit self-association but no heterotypic interactions, the system can have a three-phase region in addition to single or two-phase ones. Since each solute can phase-separate on its own, there are two binodals attached to each axis (Fig. 2E). If these binodals are large enough to meet at the center of the phase diagram, a three-phase coexisting region can emerge: single dilute phase and two distinct dense phases, each enriched with one type of solute but not the other, can form. This case highlights a possibility of multiple immiscible phases, and thus is particularly relevant to situations within the cell where multiple condensates with distinct compositions coexist. Stress granules and P-bodies are cytoplasmic condensates often contacting one another. A partial overlap in their molecular compositions and interaction networks provides an explanation for their immiscibility and adhesion (Sanders et al., 2020). Condensates themselves often exhibit multiple phases internally as can be seen in nucleoli, paraspeckles, PML bodies and stress granules (Jain et al., 2016; Lafontaine et al., 2021; Lallemand-Breitenbach and de Thé, 2018; Yamazaki et al., 2018). In general, relative magnitudes of interfacial energy impact spatial arrangements of multiphase droplets (Lu and Spruijt, 2020). Indeed, recent work provided evidence for the role of surface tension on the internal organization of condensates (Feric et al., 2016). Nucleoli exhibit a core-shell architecture consisting of FIB1-rich DFC core and NPM1-rich GC shell. Purified FIB1 and NPM1 proteins undergo phase separation to form liquid droplets, but when these two different types of droplets are combined, they stay as two immiscible phases. Consistent with the role of surface tension, hydrophobic FIB1 droplets are engulfed by hydrophilic NPM1 droplets, recapitulating nucleolar substructures.

The last case involves two solutes exhibiting both attractive homotypic and heterotypic interactions (Fig. 2F). The binodals intersect both axes, meaning that each solute can phase-separate on its own to form condensed phases. However, unlike the fourth case above where the three-phase region appears, attractive heterotypic interactions lead to mixing of condensed phases, resulting in a single condensed phase enriched with both types of solutes. Similar to this behavior, intracellular condensates are thought to form by multiple different scaffolds undergoing cooperative co-condensation. Examples of this case include components of transcriptional condensates (Cho et al., 2018). Typically, transcription factors consist of a folded DNA binding domain and a transactivation domain (Brent and Ptashne, 1985). The latter is often structurally disordered, and interacts with coactivators such as BRD4 and mediator complexes to facilitate transcription of target genes. Transcription factors such as Myc, p53, Nanog and Sox2 phase-separate to form in-vitro droplets (Boija et al., 2018). IDRs from coactivators such as MED1 and BRD4 also undergo phase separation in-vitro (Sabari et al., 2018). When transcription factors and coactivator IDRs are mixed together, two proteins cooperatively form a single dense phase enriched with both components (Boija et al., 2018). Mutations reducing intermolecular interactions between transcription factors and coactivators abrogate co-condensation. The same mutations also decrease transcriptional outputs of a reporter gene, implying a synergetic effect of co-condensation of transcriptional machineries on gene expression.

For multiple cases discussed above, ternary regular solution models provide qualitative description of diverse phase behaviors observed in biomolecular phase separation. Comparing phase separation in the ternary system to those in the binary one, it is clear that adding just another type of solute significantly increases the diversity and variability in phase behaviors. Notably, different classes of phase diagrams are a direct result of changes in intermolecular interactions. This implies that modulating interactions can be a regulatory knob that cells use to control phase behaviors. For example, tuning heterotypic interactions can turn crowders into clients or two immiscible droplets into a single droplet. The ternary model also provides important insights regarding the noise buffering effect of phase separation. Unlike the simple buffering effect in the binary system discussed above (Klosin et al., 2020), the degree of noise buffering in the ternary system varies signifi- 
cantly depending on the type of phase diagrams and the orientation of concentration variability imposed on the system (Deviri and Safran, 2021). Indeed, recent work experimentally demonstrated that the dilute-phase concentrations of intracellular condensates are often not fixed but rather increase as the expression of protein components increases (Riback et al., 2020). This behavior highlights the importance of heterotypic interactions in the formation of intracellular condensates.

\section{PHASE BEHAVIORS IN MULTICOMPONENT SYSTEMS}

Living cells contain numerous components undergoing both specific and nonspecific interactions with one another. Considering diverse types of phase diagrams appeared in the ternary system, it is not unreasonable to expect even higher levels of diversity in intracellular phase behavior. At first sight, the complexity of multicomponent phase behaviors appears intractable. However, several works have begun to bridge the gap between simple systems consisting of a few components on one hand, and multicomponent mixtures such as living cells (Jacobs and Frenkel, 2013; 2017). To model an N-component system, these works took a statistical approach, originally pioneered by Sear and Cuesta (2003), where pair-wise interactions between molecules are randomly drawn from a single Gaussian distribution. A major conclusion of the model is that the width of Gaussian distribution, i.e., variability in intermolecular interactions, is an important parameter distinguishing between two different types of phase separation: condensation and demixing. The former involves two coexisting phases with nearly identical compositions but differing in total concentration of molecules. In the latter scenario, a few species demix from others, forming phases with distinct compositions. These works suggest that tuning a few intermolecular interactions can induce the formation of demixed phases, and thus theoretically support the presence of diverse condensates in living cells.

A key question concerns how stability, miscibility and composition of individual condensates with multiple components are defined. Recent patchy-colloid based modeling suggested that the molecular connectivity in the multicomponent dense phase is a key parameter determining the stability and composition of condensates (Espinosa et al., 2020). Thus, the valency of individual components and the type of interaction nodes each component makes with others can shape together the phase diagram of specific condensates. Using stress granules and P-bodies as a model system, recent experimental work further elaborated this concept and emphasized the role of competing interaction nodes in tuning the phase miscibility (Sanders et al., 2020).

\section{CONCLUSION}

Phase separation is a physicochemical phenomenon driving a well-mixed solution into coexisting phases with distinct compositions. For the past few years, extensive studies have been carried out on diverse phase behaviors of biomolecules, and uncovered the role of phase separation in intracellular organization. The idea of phase separation has promoted our understanding of mechanisms by which cells regulate the assembly, composition and substructures of condensates.
However, it must also be noted that experimental evidence for phase separation within living cells is often descriptive and lacks quantitative nature (McSwiggen et al., 2019), leaving a gap between phase behaviors observed with purified protein components and those of intracellular condensates. The model-based quantitative analysis of experimental data from living cells can help bridge this gap by providing predictive features that can be tested. This approach can then be fed back into the modeling process, which can ultimately provide important insights in the way intracellular phases behave (Riback et al., 2020).

In this regard, we discussed recent progress on biomolecular phase separation in light of regular solution models of binary and ternary systems. We showed that even a simple binary model can explain key biophysical features of intracellular condensates such as the presence of saturation concentrations and noise buffering. The ternary regular solution model highlights that heterotypic interactions provided by extra components can give rise to much richer phase behaviors including the change in saturation concentrations, re-entrant transitions and composition controls. Notably, despite being highly useful in describing the overall behaviors of many phase-separating systems, regular solution models have a limited capacity to predict quantitative details such as precise locations of phase boundary. In general, future work will be necessary to shed more light on the relation between the sequences of proteins and phase behaviors they exhibit. For example, little is known about how sequence features determine the phase immiscibility in multiphase systems. More work is also needed to elucidate how crowded intracellular environment would modulate phase separation properties. The model-based studies of biomolecular phase separation will be essential to systematically probe full complexity of intracellular organization.

\section{ACKNOWLEDGMENTS}

This work is supported by the National Research Foundation (NRF) of Korea (NRF-2019R1C1C1006477), the National Convergence Research of Scientific Challenges (NRF2020M3F7A1094300), and Creative-Pioneering Researchers Program through Seoul National University.

\section{CONFLICT OF INTEREST}

The author has no potential conflicts of interest to disclose.

\section{ORCID}

Yongdae Shin https://orcid.org/0000-0003-3441-1986

\section{REFERENCES}

Aumiller, W.M., Jr. and Keating, C.D. (2016). Phosphorylation-mediated RNA/peptide complex coacervation as a model for intracellular liquid organelles. Nat. Chem. 8, 129-137.

Banani, S.F., Rice, A.M., Peeples, W.B., Lin, Y., Jain, S., Parker, R., and Rosen, M.K. (2016). Compositional control of phase-separated cellular bodies. Cell 166, 651-663.

Banani, S.F., Lee, H.O., Hyman, A.A., and Rosen, M.K. (2017). Biomolecular condensates: organizers of cellular biochemistry. Nat. Rev. Mol. Cell Biol. $18,285-298$. 
Banerjee, P.R., Milin, A.N., Moosa, M.M., Onuchic, P.L., and Deniz, A.A. (2017). Reentrant phase transition drives dynamic substructure formation in ribonucleoprotein droplets. Angew. Chem. Int. Ed. Engl. 56, 1135411359.

Basu, S., Mackowiak, S.D., Niskanen, H., Knezevic, D., Asimi, V., Grosswendt, S., Geertsema, H., Ali, S., Jerković, I., Ewers, H., et al. (2020). Unblending of transcriptional condensates in human repeat expansion disease. Cell 181, 1062-1079.e30.

Benedek, G.B. (1997). Cataract as a protein condensation disease: the Proctor Lecture. Invest. Ophthalmol. Vis. Sci. 38, 1911-1921.

Boija, A., Klein, I.A., Sabari, B.R., Dall'Agnese, A., Coffey, E.L., Zamudio, A.V., Li, C.H., Shrinivas, K., Manteiga, J.C., Hannett, N.M., et al. (2018). Transcription factors activate genes through the phase-separation capacity of their activation domains. Cell 175, 1842-1855.e16.

Bracha, D., Walls, M.T., Wei, M.T., Zhu, L., Kurian, M., Avalos, J.L., Toettcher, J.E., and Brangwynne, C.P. (2018). Mapping local and global liquid phase behavior in living cells using photo-oligomerizable seeds. Cell 175, 14671480.e13.

Brangwynne, C., Mitchison, T., and Hyman, A.A. (2011). Active liquid-like behavior of nucleoli determines their size and shape in Xenopus laevis oocytes. Proc. Natl. Acad. Sci. U. S. A. 108, 4334-4339.

Brangwynne, C.P., Eckmann, C.R., Courson, D.S., Rybarska, A., Hoege, C., Gharakhani, J., Jülicher, F., and Hyman, A.A. (2009). Germline P granules are liquid droplets that localize by controlled dissolution/condensation. Science 324, 1729-1732.

Brangwynne, C.P., Tompa, P., and Pappu, R.V. (2015). Polymer physics of intracellular phase transitions. Nat. Phys. 11, 899-904.

Brent, R. and Ptashne, M. (1985). A eukaryotic transcriptional activator bearing the DNA specificity of a prokaryotic repressor. Cell 43(3 Pt 2), 729-736.

Broide, M.L., Berland, C.R., Pande, J., Ogun, O.O., and Benedek, G.B. (1991). Binary-liquid phase-separation of lens protein solutions. Proc. Natl. Acad. Sci. U. S. A. 88, 5660-5664.

Buchan, J.R. and Parker, R. (2009). Eukaryotic stress granules: the ins and outs of translation. Mol. Cell 36, 932-941.

Burke, K.A., Janke, A.M., Rhine, C.L., and Fawzi, N.L. (2015). Residue-byresidue view of in vitro FUS granules that bind the C-terminal domain of RNA polymerase II. Mol. Cell 60, 231-241.

Cai, D., Feliciano, D., Dong, P., Flores, E., Gruebele, M., Porat-Shliom, N., Sukenik, S., Liu, Z., and Lippincott-Schwartz, J. (2019). Phase separation of YAP reorganizes genome topology for long-term YAP target gene expression. Nat. Cell Biol. 21, 1578-1589.

Chen, D. and Huang, S. (2001). Nucleolar components involved in ribosome biogenesis cycle between the nucleolus and nucleoplasm in interphase cells. J. Cell Biol. 153, 169-176.

Chen, X., Wu, X., Wu, H., and Zhang, M. (2020). Phase separation at the synapse. Nat. Neurosci. 23, 301-310.

Cho, W.K., Spille, J.H., Hecht, M., Lee, C., Li, C., Grube, V., and Cisse, I.I. (2018). Mediator and RNA polymerase II clusters associate in transcription-dependent condensates. Science 361, 412-415.

Choi, J.M., Holehouse, A.S., and Pappu, R.V. (2020). Physical principles underlying the complex biology of intracellular phase transitions. Annu. Rev. Biophys. 49, 107-133.

Chujo, T. and Hirose, T. (2017). Nuclear bodies built on architectural long noncoding RNAs: unifying principles of their construction and function. Mol. Cells 40, 889-896.

Deviri, D. and Safran, S.A. (2021). Physical theory of biological noise buffering by multicomponent phase separation. Proc. Natl. Acad. Sci. U. S. A. 118, e2100099118.

Dignon, G.L., Best, R.B., and Mittal, J. (2020). Biomolecular phase separation: from molecular driving forces to macroscopic properties.
Annu. Rev. Phys. Chem. 71, 53-75.

Dormann, D., Rodde, R., Edbauer, D., Bentmann, E., Fischer, I., Hruscha, A., Than, M.E., Mackenzie, I.R.A., Capell, A., Schmid, B., et al. (2010). ALS-associated fused in sarcoma (FUS) mutations disrupt Transportinmediated nuclear import. EMBO J. 29, 2841-2857.

Elbaum-Garfinkle, S., Kim, Y., Szczepaniak, K., Chen, C.C.H., Eckmann, C.R. Myong, S., and Brangwynne, C.P. (2015). The disordered P granule protein LAF-1 drives phase separation into droplets with tunable viscosity and dynamics. Proc. Natl. Acad. Sci. U. S. A. 112, 7189-7194.

Espinosa, J.R., Joseph, J.A., Sanchez-Burgos, I., Garaizar, A., Frenkel, D., and Collepardo-Guevara, R. (2020). Liquid network connectivity regulates the stability and composition of biomolecular condensates with many components. Proc. Natl. Acad. Sci. U. S. A. 117, 13238-13247.

Feric, M., Vaidya, N., Harmon, T.S., Mitrea, D.M., Zhu, L., Richardson, T.M., Kriwacki, R.W., Pappu, R.V., and Brangwynne, C.P. (2016). Coexisting liquid phases underlie nucleolar subcompartments. Cell 165, 1686-1697.

Franzmann, T.M., Jahnel, M., Pozniakovsky, A., Mahamid, J., Holehouse, A.S., Nüske, E., Richter, D., Baumeister, W., Grill, S.W., Pappu, R.V., et al. (2018). Phase separation of a yeast prion protein promotes cellular fitness. Science 359, eaao5654.

Freeman Rosenzweig, E.S., Xu, B., Kuhn Cuellar, L., Martinez-Sanchez, A., Schaffer, M., Strauss, M., Cartwright, H.N., Ronceray, P., Plitzko, J.M., Förster, F., et al. (2017). The eukaryotic $\mathrm{CO}_{2}$-concentrating organelle is liquid-like and exhibits dynamic reorganization. Cell 171, 148-162.e19.

Guillén-Boixet, J., Kopach, A., Holehouse, A.S., Wittmann, S., Jahnel, M., Schlüßler, R., Kim, K., Trussina, I.R.E.A., Wang, J., Mateju, D., et al. (2020). RNA-induced conformational switching and clustering of G3BP drive stress granule assembly by condensation. Cell 181, 346-361.e17.

Harmon, T.S., Holehouse, A.S., Rosen, M.K., and Pappu, R.V. (2017). Intrinsically disordered linkers determine the interplay between phase separation and gelation in multivalent proteins. Elife 6, e30294.

Henninger, J.E., Oksuz, O., Shrinivas, K., Sagi, I., LeRoy, G., Zheng, M.M. Andrews, J.O., Zamudio, A.V., Lazaris, C., Hannett, N.M., et al. (2021). RNAmediated feedback control of transcriptional condensates. Cell 184, 207225.e24.

Hofweber, M., Hutten, S., Bourgeois, B., Spreitzer, E., Niedner-Boblenz, A., Schifferer, M., Ruepp, M.D., Simons, M., Niessing, D., Madl, T., et al. (2018). Phase separation of FUS is suppressed by its nuclear import receptor and arginine methylation. Cell 173, 706-719.e13.

Hong, S., Choi, S., Kim, R., and Koh, J. (2020). Mechanisms of macromolecular interactions mediated by protein intrinsic disorder. Mol. Cells 43, 899-908.

Ishimoto, C. and Tanaka, T. (1977). Critical behavior of a binary mixture of protein and salt water. Phys. Rev. Lett. 39, 474-477.

Jacobs, W.M. and Frenkel, D. (2013). Predicting phase behavior in multicomponent mixtures. J. Chem. Phys. 139, 024108.

Jacobs, W.M. and Frenkel, D. (2017). Phase transitions in biological systems with many components. Biophys. J. 112, 683-691.

Jain, S., Wheeler, J.R., Walters, R.W., Agrawal, A., Barsic, A., and Parker, R. (2016). ATPase-modulated stress granules contain a diverse proteome and substructure. Cell 164, 487-498.

Kanaan, N.M., Hamel, C., Grabinski, T., and Combs, B. (2020). Liquid-liquid phase separation induces pathogenic tau conformations in vitro. Nat. Commun. 11, 2809.

Kato, M., Han, T.W., Xie, S., Shi, K., Du, X., Wu, L.C., Mirzaei, H., Goldsmith, E.J., Longgood, J., Pei, J., et al. (2012). Cell-free formation of RNA granules: low complexity sequence domains form dynamic fibers within hydrogels. Cell 149, 753-767.

Klosin, A., Oltsch, F., Harmon, T., Honigmann, A., Jülicher, F., Hyman, A.A., and Zechner, C. (2020). Phase separation provides a mechanism to reduce noise in cells. Science 367, 464-468. 
Lafontaine, D.L.J., Riback, J.A., Bascetin, R., and Brangwynne, C.P. (2021). The nucleolus as a multiphase liquid condensate. Nat. Rev. Mol. Cell Biol. $22,165-182$

Lallemand-Breitenbach, V. and de Thé, H. (2018). PML nuclear bodies: from architecture to function. Curr. Opin. Cell Biol. 52, 154-161.

Li, P., Banjade, S., Cheng, H., Kim, S., Chen, B., Guo, L., Llaguno, M., Hollingsworth, J.V., King, D.S., Banani, S.F., et al. (2012). Phase transitions in the assembly of multivalent signalling proteins. Nature 483, 336-340.

Lin, Y.H., Forman-Kay, J.D., and Chan, H.S. (2016). Sequence-specific polyampholyte phase separation in membraneless organelles. Phys. Rev. Lett. 117, 178101.

Lin, Y., Protter, D.S.W., Rosen, M.K., and Parker, R. (2015). Formation and maturation of phase-separated liquid droplets by RNA-binding proteins. Mol. Cell 60, 208-219.

Lu, T. and Spruijt, E. (2020). Multiphase complex coacervate droplets. J. Am. Chem. Soc. 142, 2905-2914.

Maharana, S., Wang, J., Papadopoulos, D.K., Richter, D., Pozniakovsky, A., Poser, I., Bickle, M., Rizk, S., Guillén-Boixet, J., Franzmann, T., et al. (2018). RNA buffers the phase separation behavior of prion-like RNA binding proteins. Science 360, 918-921.

Mathieu, C., Pappu, R.V., and Taylor, J.P. (2020). Beyond aggregation: pathological phase transitions in neurodegenerative disease. Science 370 , 56-60.

Mao, Y.S., Zhang, B., and Spector, D.L. (2011). Biogenesis and function of nuclear bodies. Trends Genet. 27, 295-306.

McSwiggen, D.T., Mir, M., Darzacq, X., and Tjian, R. (2019). Evaluating phase separation in live cells: diagnosis, caveats, and functional consequences. Genes Dev. 33, 1619-1634.

Meijering, J.L. (1950). Segregation in regular ternary solutions. Philips Res. Rep. 5, 335-356.

Milo, R. (2013). What is the total number of protein molecules per cell volume? A call to rethink some published values. Bioessays 35, 10501055.

Mollet, S., Cougot, N., Wilczynska, A., Dautry, F., Kress, M., Bertrand, E., and Weil, D. (2008). Translationally repressed mRNA transiently cycles through stress granules during stress. Mol. Biol. Cell 19, 4469-4479.

Molliex, A., Temirov, J., Lee, J., Coughlin, M., Kanagaraj, A.P., Kim, H.J., Mittag, T., and Taylor, J.P. (2015). Phase separation by low complexity domains promotes stress granule assembly and drives pathological fibrillization. Cell 163, 123-133.

Monahan, Z., Ryan, V.H., Janke, A.M., Burke, K.A., Rhoads, S.N., Zerze, G.H., O'Meally, R., Dignon, G.L., Conicella, A.E., Zheng, W., et al. (2017). Phosphorylation of the FUS low-complexity domain disrupts phase separation, aggregation, and toxicity. EMBO J. 36, 2951-2967.

Murakami, T., Qamar, S., Lin, J.Q., Schierle, G.S., Rees, E., Miyashita, A., Costa, A.R., Dodd, R.B., Chan, F.T., Michel, C.H., et al. (2015). ALS/FTD mutation-induced phase transition of FUS liquid droplets and reversible hydrogels into irreversible hydrogels impairs RNP granule function. Neuron 88, 678-690.

Murthy, A.C., Dignon, G.L., Kan, Y., Zerze, G.H., Parekh, S.H., Mittal, J., and Fawzi, N.L. (2019). Molecular interactions underlying liquid-liquid phase separation of the FUS low-complexity domain. Nat. Struct. Mol. Biol. 26, 637-648.

Nott, T.J., Petsalaki, E., Farber, P., Jervis, D., Fussner, E., Plochowietz, A., Craggs, T.D., Bazett-Jones, D.P., Pawson, T., Forman-Kay, J.D., et al. (2015). Phase transition of a disordered nuage protein generates environmentally responsive membraneless organelles. Mol. Cell 57, 936-947.

Nott, T.J., Craggs, T.D., and Baldwin, A.J. (2016). Membraneless organelles can melt nucleic acid duplexes and act as biomolecular filters. Nat. Chem. $8,569-575$.

Pak, C.W., Kosno, M., Holehouse, A.S., Padrick, S.B., Mittal, A., Ali, R., Yunus,
A.A., Liu, D.R., Pappu, R.V., and Rosen, M.K. (2016). Sequence determinants of intracellular phase separation by complex coacervation of a disordered protein. Mol. Cell 63, 72-85.

Patel, A., Lee, H.O., Jawerth, L., Maharana, S., Jahnel, M., Hein, M.Y., Stoynov, S., Mahamid, J., Saha, S., Franzmann, T.M., et al. (2015). A liquidto-solid phase transition of the ALS protein FUS accelerated by disease mutation. Cell 162, 1066-1077.

Peeples, W. and Rosen, M.K. (2021). Mechanistic dissection of increased enzymatic rate in a phase-separated compartment. Nat. Chem. Biol. 17, 693-702.

Posey, A.E., Holehouse, A.S., and Pappu, R.V. (2018). Phase separation of intrinsically disordered proteins. Methods Enzymol. 611, 1-30.

Qamar, S., Wang, G.Z., Randle, S.J., Ruggeri, F.S., Varela, J.A., Lin, J.Q., Phillips, E.C., Miyashita, A., Williams, D., Ströhl, F., et al. (2018). FUS phase separation is modulated by a molecular chaperone and methylation of arginine cation- $\pi$ interactions. Cell 173, 720-734.e15.

Rai, A.K., Chen, J., Selbach, M., and Pelkmans, L. (2018). Kinase-controlled phase transition of membraneless organelles in mitosis. Nature 559, 211216.

Ray, S., Singh, N., Kumar, R., Patel, K., Pandey, S., Datta, D., Mahato, J., Panigrahi, R., Navalkar, A., Mehra, S., et al. (2020). $\alpha$-Synuclein aggregation nucleates through liquid-liquid phase separation. Nat. Chem. 12, 705-716.

Riback, J.A., Zhu, L., Ferrolino, M.C., Tolbert, M., Mitrea, D.M., Sanders, D.W., Wei, M., Kriwacki, R.W., and Brangwynne, C.P. (2020). Compositiondependent thermodynamics of intracellular phase separation. Nature 581, 209-214.

Rubinstein, M. and Colby, R.H. (2003). Polymer Physics (Oxford: Oxford University Press).

Ruff, K.M., Dar, F., and Pappu, R.V. (2021). Ligand effects on phase separation of multivalent macromolecules. Proc. Natl. Acad. Sci. U. S. A. 118, e2017184118.

Ryan, V.H., Dignon, G.L., Zerze, G.H., Chabata, C.V., Silva, R., Conicella, A.E., Amaya, J., Burke, K.A., Mittal, J., and Fawzi, N.L. (2018). Mechanistic view of hnRNPA2 low-complexity domain structure, interactions, and phase separation altered by mutation and arginine methylation. Mol. Cell 69, 465-479.e7.

Sabari, B.R., Dall'Agnese, A., Boija, A., Klein, I.A., Coffey, E.L., Shrinivas, K., Abraham, B.J., Hannett, N.M., Zamudio, A.V., Manteiga, J.C., et al. (2018). Coactivator condensation at super-enhancers links phase separation and gene control. Science 361, eaar3958.

Sanders, D.W., Kedersha, N., Lee, D.S.W., Strom, A.R., Drake, V., Riback, J.A., Bracha, D., Eeftens, J.M., Iwanicki, A., Wang, A., et al. (2020). Competing protein-RNA interaction networks control multiphase intracellular organization. Cell 181, 306-324.e28.

Sear, R.P. and Cuesta, J.A. (2003). Instabilities in complex mixtures with a large number of components. Phys. Rev. Lett. 91, 245701.

Shin, Y. and Brangwynne, C.P. (2017). Liquid phase condensation in cell physiology and disease. Science 357, eaaf4382.

Shin, Y., Berry, J., Pannucci, N., Haataja, M.P., Toettcher, J.E., and Brangwynne, C.P. (2017). Spatiotemporal control of intracellular phase transitions using light-activated optoDroplets. Cell 168, 159-171.e14.

Simon, J.R., Carroll, N.J., Rubinstein, M., Chilkoti, A., and López, G.P. (2017). Programming molecular self-assembly of intrinsically disordered proteins containing sequences of low complexity. Nat. Chem. 9, 509-515.

Snead, W.T. and Gladfelter, A.S. (2019). The control centers of biomolecular phase separation: how membrane surfaces, PTMs, and active processes regulate condensation. Mol. Cell 76, 295-305.

Strom, A.R., Emelyanov, A.V., Mir, M., Fyodorov, D.V., Darzacq, X., and Karpen, G.H. (2017). Phase separation drives heterochromatin domain formation. Nature 547, 241-245.

Su, X., Ditlev, J.A., Hui, E., Xing, W., Banjade, S., Okrut, J., King, D.S., Taunton, 
J., Rosen, M.K., and Vale, R.D. (2016). Phase separation of signaling molecules promotes T cell receptor signal transduction. Science 352, 595599.

Thomson, J.A., Schurtenberger, P., Thurston, G.M., and Benedek, G.B. (1987). Binary liquid phase separation and critical phenomena in a protein/water solution. Proc. Natl. Acad. Sci. U. S. A. 84, 7079-7083.

Thul, P.J., Akesson, L., Wiking, M., Mahdessian, D., Geladaki, A., Ait Blal, H., Alm, T., Asplund, A., Björk, L., Breckels, L.M., et al. (2017). A subcellular map of the human proteome. Science 356, eaal3321.

Tompa, P. (2012). Intrinsically disordered proteins: a 10-year recap. Trends Biochem. Sci. 37, 509-516.

Ukmar-Godec, T., Hutten, S., Grieshop, M.P., Rezaei-Ghaleh, N., CimaOmori, M.S., Biernat, J., Mandelkow, E., Söding, J., Dormann, D., and Zweckstetter, M. (2019). Lysine/RNA-interactions drive and regulate biomolecular condensation. Nat. Commun. 10, 2909.

Uversky, V.N. (2017). Intrinsically disordered proteins in overcrowded milieu: membrane-less organelles, phase separation, and intrinsic disorder. Curr. Opin. Struct. Biol. 44, 18-30.

Uversky, V.N. (2021). Recent developments in the field of intrinsically disordered proteins: intrinsic disorder-based emergence in cellular biology in light of the physiological and pathological liquid-liquid phase transitions. Annu. Rev. Biophys. 50, 135-156.

Wang, J., Choi, J.M., Holehouse, A.S., Lee, H.O., Zhang, X., Jahnel, M., Maharana, S., Lemaitre, R., Pozniakovsky, A., Drechsel, D., et al. (2018). A molecular grammar governing the driving forces for phase separation of prion-like RNA binding proteins. Cell 174, 688-699.e16.

Weber, S.C. and Brangwynne, C.P. (2015). Inverse size scaling of the nucleolus by a concentration-dependent phase transition. Curr. Biol. 25, 641-646.

Wei, M.T., Elbaum-Garfinkle, S., Holehouse, A.S., Chen, C.C.H., Feric, M., Arnold, C.B., Priestley, R.D., Pappu, R.V., and Brangwynne, C.P. (2017). Phase behaviour of disordered proteins underlying low density and high permeability of liquid organelles. Nat. Chem. 9, 1118-1125.

Wei, M.T., Chang, Y.C., Shimobayashi, S.F., Shin, Y., Strom, A.R., and Brangwynne, C.P. (2020). Nucleated transcriptional condensates amplify gene expression. Nat. Cell Biol. 22, 1187-1196.

Weidtkamp-Peters, S., Lenser, T., Negorev, D., Gerstner, N., Hofmann, T.G., Schwanitz, G., Hoischen, C., Maul, G., Dittrich, P., and Hemmerich, P.
(2008). Dynamics of component exchange at PML nuclear bodies. J. Cell Sci. 121, 2731-2743.

Wiedner, H.J. and Giudice, J. (2021). It's not just a phase: function and characteristics of RNA-binding proteins in phase separation. Nat. Struct. Mol. Biol. 28, 465-473.

Wilfling, F., Lee, C.W., Erdmann, P.S., Zheng, Y., Sherpa, D., Jentsch, S., Pfander, B., Schulman, B.A., and Baumeister, W. (2020). A selective autophagy pathway for phase-separated endocytic protein deposits. Mol. Cell 80, 764-778.e7.

Yamazaki, T., Souquere, S., Chujo, T., Kobelke, S., Chong, Y.S., Fox, A.H., Bond, C.S., Nakagawa, S., Pierron, G., and Hirose, T. (2018). Functional domains of NEAT1 architectural IncRNA induce paraspeckle assembly through phase separation. Mol. Cell 70, 1038-1053.e7.

Yang, P., Mathieu, C., Kolaitis, R.M., Zhang, P., Messing, J., Yurtsever, U., Yang, Z., Wu, J., Li, Y., Pan, Q., et al. (2020). G3BP1 is a tunable switch that triggers phase separation to assemble stress granules. Cell 181, 325-345. e28.

Yasuda, S., Tsuchiya, H., Kaiho, A., Guo, Q., Ikeuchi, K., Endo, A., Arai, N., Ohtake, F., Murata, S., Inada, T., et al. (2020). Stress- and ubiquitylationdependent phase separation of the proteasome. Nature 578, 296-300.

Zamudio, A.V., Dall'Agnese, A., Henninger, J.E., Manteiga, J.C., Afeyan, L.K., Hannett, N.M., Coffey, E.L., Li, C.H., Oksuz, O., Sabari, B.R., et al. (2019). Mediator condensates localize signaling factors to key cell identity genes. Mol. Cell 76, 753-766.e6.

Zeng, M., Shang, Y., Araki, Y., Guo, T., Huganir, R.L., and Zhang, M. (2016). Phase transition in postsynaptic densities underlies formation of synaptic complexes and synaptic plasticity. Cell 166, 1163-1175.e12.

Zhang, G., Wang, Z., Du, Z., and Zhang, H. (2018). mTOR regulates phase separation of $\mathrm{PGL}$ granules to modulate their autophagic degradation. Cell 174, 1492-1506.e22.

Zhang, J.Z., Lu, T.W., Stolerman, L.M., Tenner, B., Yang, J.R., Zhang, J.F., Falcke, M., Rangamani, P., Taylor, S.S., Mehta, S., et al. (2020). Phase separation of a PKA regulatory subunit controls CAMP compartmentation and oncogenic signaling. Cell 182, 1531-1544.e15.

Zhu, L., Richardson, T.M., Wacheul, L., Wei, M.T., Feric, M., Whitney, G., Lafontaine, D.L.J., and Brangwynne, C.P. (2019). Controlling the material properties and rRNA processing function of the nucleolus using light. Proc. Natl. Acad. Sci. U. S. A. 116, 17330-17335. 